\title{
THE SOLUTION OF FRACTIONAL NONLINEAR GINZBURG-LANDAU EQUATION WITH WEAK INITIAL DATA
}

\author{
Jie Xin, JiAqian Hu And Hong Lu
}

Abstract. In this paper, we study the solution of the fractional nonlinear Ginzburg-Landau(FNGL) equation with weak initial data in the weighted Lebesgue spaces. The existence of a solution to this equation is proved by the contraction-mapping principle.

Mathematics subject classification (2010): 26A33, 35R11, 35Q56.

Keywords and phrases: Fractional nonlinear Ginzburg-Landau equation, global solution, contractionmapping principle, weighted Lebesgue spaces.

\section{REFERENCES}

[1] H. WeitZner And G. M. Zaslavs Ky, Some applications of fractional equations, Commun. Nonlinear Sci. Numer Simul 8 (2003), 273-281.

[2] Vasily E. Tarasov and George M. Zaslavsky, Fractional Ginzburg-Landau equation for fractal media, Physica A 354 (2005), 247-261.

[3] A. V. Milovanov and J. Tuul Rasmussen, Fractional generalization of the Ginzburg-Landau equation: an unconventional approach to critical phenomena in complex media, Physics Letters A 337 (2005), 75-80.

[4] J.-H. Wu, Quasi-geostrophic type equations with weak initial data, Electon. J. Differential Equations (1998), 1-10.

[5] Carlos E. Kenig, Gustavo Ponce And Luis Vega, Well-posedness and scattering results for the generalized Korteweg-de Vries equation via the contraction principle, Comm. Pure Appl. Math. XLVI (1993), 527-620.

[6] T. Kato And G. Ponce, Commutator estimates and the Euler and Navier-Stokes equations, Comm. Pure Appl. Math. XXI (1998), 891-907.

[7] R. R. Coifman And Y. MeYer, Nonlinear harmonic analysis, operator theory and P.D.E., in "Beijing Lectures in Harmonic Analysis", Princeton University Press (1986), 3-45.

[8] T. Kato And G. Ponce, The Navier-Stokes equations with weak initial data, Int Math. Research Notices 10 (1994), 435-444.

[9] Y. Giga, T. Miyakawa AND H. OsadA, Two dimensional Navier-Stokes flow with measures as initial vorticity, Arch. Rotional Mech. Anal. 104 (1998), 223-250. 